\title{
HIV Treatment in the Criminal Justice System: Critical Knowledge and Intervention Gaps
}

\author{
Jaimie P. Meyer, Nadine E. Chen, and Sandra A. Springer \\ AIDS Care Program, Infectious Diseases Section, Yale University School of Medicine, 135 College Street, Suite 323, New Haven, \\ CT 06510-2283, USA \\ Correspondence should be addressed to Sandra A. Springer, sandra.springer@yale.edu
}

Received 24 December 2010; Revised 10 April 2011; Accepted 5 May 2011

Academic Editor: Magnus Gisslén

Copyright () 2011 Jaimie P. Meyer et al. This is an open access article distributed under the Creative Commons Attribution License, which permits unrestricted use, distribution, and reproduction in any medium, provided the original work is properly cited.

\begin{abstract}
The criminal justice system bears a disproportionate burden of the HIV epidemic. Continuity of care is critical for HAART-based prevention of HIV-related morbidity and mortality. This paper describes four major challenges to successful management of HIV in the criminal justice system: relapse to substance use, homelessness, mental illness, and loss of medical and social benefits. Each of these areas constitutes a competing priority upon release that demands immediate attention and diverts time, energy, and valuable resources away from engagement in care and adherence to HAART. Numerous gaps exist in scientific knowledge about these issues and potential solutions. In illuminating these knowledge deficits, we present a contemporary research agenda for the management of HIV in correctional systems. Future empirical research should focus on these critical issues in HIV-infected prisoners and releasees while interventional research should incorporate evidence-based solutions into the criminal justice setting.
\end{abstract}

\section{Introduction}

The criminal justice setting provides vast opportunities for early diagnosis, prevention, and treatment of HIV $[1,2]$. One in seven people living with HIV in the United States passes through the criminal justice system (CJS) each year [3], and incarceration is considered an independent risk factor for HIV infection [4]. For those living with HIV, history of incarceration is a strong predictor of nonadherence to HIV treatment and care $[5,6]$.

Correctional facilities thus bear a disproportionate burden of the HIV epidemic in the USA with a prevalence rate of HIV 3-5 times higher than surrounding communities $[3,7]$. Though substantial need exists for management of HIV during incarceration, it is complicated by equally prevalent comorbid medical and psychiatric diseases, [8] potential lack of privacy around HIV testing and treatment, [9] inmates' frequent mistrust of the healthcare system, [10] and issues of control related to the prison environment itself [11]. Perhaps as a result, only an estimated one-third of HIV-infected inmates with a clinical indication for therapy receive combination antiretroviral therapy (HAART) during incarceration $[12,13]$.

Despite these obstacles, management of HIV in correctional settings has been shown to be feasible, acceptable, [10] and highly successful [7]. In Connecticut, an impressive $59 \%$ of HIV-infected inmates achieved an undetectable viral load (i.e., $<400$ copies $/ \mathrm{mL}$ ) by the end of their incarceration [14]. In this incarcerated cohort, clinical benefit was derived regardless of the type of HAART regimen prescribed [15]. Equal access to health care during incarceration also enables impoverished and minority subpopulations to overcome some healthcare disparities that exist outside prison walls $[13,16]$. The highly structured nature of prison contributes to these successes, providing a relatively drug-free environment and granting opportunities to optimize medication adherence through directly administered antiretroviral therapy [17]. The provision of HAART in USA correctional facilities is also supported by a Supreme Court decision that established there cannot be "deliberate indifference" to the health needs of inmates [18] and by state laws that protect the rights to care of HIV-infected inmates in particular [19]. 
Unfortunately, benefits of prison-based HAART are rarely sustained upon return to communities [14, 20, 21]. In the Texas CJS, few prisoners filled prescriptions for their HIV medications [22] or enrolled in an HIV clinic within 30 days following release [23]. Upon release from prison, individuals face difficulties adhering to HAART and HIV-related care services because they are often distracted by basic subsistence needs and temptations of drug relapse [24]. Medication nonadherence is associated with serious negative individual and public health consequences. Although even intermittent HAART adherence may provide some immunologic or virologic benefit, [25] the Strategies for Management of Antiretroviral Therapy (SMART) study demonstrated that continuous therapy has superior long-term outcomes in terms of AIDS-related morbidity and mortality [26].

There is a paucity of data evaluating barriers to sustained antiretroviral therapy for $\mathrm{HIV}$-infected persons transitioning from the CJS to the community. In this review, we outline major obstacles to continuous HIV care among populations who interface with the CJS, both during incarceration and after release. Major challenges include relapse to substance use, housing instability, comorbid mental illness, and coverage gaps in medical and social benefits. We briefly describe the significance of each of these issues in general CJS or in HIV-infected nonincarcerated populations and then explore potential areas for future empirical and interventional research. Rigorous scientific evaluation of these "knowledge gaps" is critical for progress in the field. Furthermore, success of programs designed to facilitate transition between prisons and communities hinges on bringing evidence-based solutions into the criminal justice setting.

\section{Methods}

A search strategy was undertaken using PubMed, OvidSP, and MEDLINE. The following key terms were used: HIV, HAART, adherence, outcomes, prisoners, prison/jail, incarcerated, inmates, criminal justice, corrections, transition, linkage, and released. These were combined with terms relevant to each topic of interest: substance, addiction, drugs, alcohol, dependence/use/abuse, homelessness, housing, mental illness, depression, insurance, Medicaid, and benefits. Continuity of care was defined in terms of HAART adherence and enrollment in HIV care upon release. Additional references from seminal papers were reviewed to broaden the search and assure that important contributions were not overlooked. Articles were included if they were written in English and were published in peer-reviewed journals between 19902011.

\section{Results}

\subsection{Relapse to Substance Use}

3.1.1. Epidemiology of the Problem. Drug use and crime are inextricably linked. In the United States, where the "war on drugs" has devolved into a "war on drug users," [27] drugrelated offenses are punishable by incarceration. As a result, the USA, which contributes only $5 \%$ of the world population, holds $25 \%$ of the world prisoners [28]. In this context, almost half of USA prisoners screen positive for marijuana, cocaine, heroin, or methamphetamine at the time of their arrest [29], and $25 \%$ of violent offenders are under the influence of drugs and alcohol when they commit crimes [30]. The CJS bears a disproportionate burden of the epidemic of addiction, with up to $65 \%$ of prison inmates meeting DSM-IV criteria for drug or alcohol abuse or addiction [28, 30, 31]. In jails, in which inmates are either unsentenced or sentenced to shorter terms, women report even higher rates of substance use disorders (SUDs) than their male counterparts [32]. Up to $70 \%$ of HIV-infected prisoners meet criteria for opioid dependence [33]. These ongoing and often untreated SUDs play a major role in prison recidivism and likely contribute to poor HIV outcomes after release.

3.1.2. Areas for Future Empirical Research. SUDs have a seemingly profound impact on the health outcomes of HIV-infected prisoners, for whom addiction-related lapses in HAART continuity threaten virologic suppression after release. With some notable exceptions described here, there have been relatively few published studies regarding the effect of SUDs on HIV outcomes in released prisoners. A 2008 study of HIV-infected reincarcerated jail detainees examined HAART adherence between the time of prison release and reincarceration. Findings revealed that those subjects who discontinued HAART were three times more likely to have also relapsed to marijuana or injection drug use between incarcerations [34]. Among HIV-infected injection drug users in Vancouver, any alcohol use or incarceration in the prior six months was associated with poor HAART adherence and worse virologic outcomes [35]. In a smaller study of 30 HIV-infected recently released prisoners, only 18 (60\%) were enrolled in HIV primary care 21 days after incarceration. Enrollment in primary care was associated with abstinence from alcohol though this association did not reach statistical significance, likely owing to the small sample size [36]. Other published research on reduced uptake in HIV care after prison release has not assessed the important contribution of SUDs to discontinuous care $[22,23]$.

Extrapolating from studies of community-based subjects with HIV outside of the CJS, alcohol use is associated with decreased initiation of and adherence to HAART as well as increased rates of virologic failure [37]. Similarly, relapse to drug use, especially injection drugs and crack cocaine, has been associated with decreased HAART adherence, decreased engagement in HIV care, and increased risk of developing AIDS-defining conditions [5, 38]. The field would benefit from parallel research on the effect of SUDs on HIV biologic outcomes among prisoners and releasees living with HIV.

3.1.3. Areas for Future Interventional Research. Despite overwhelming demonstrated need, substance abuse treatment programs during incarceration are relatively nonexistent [39]. As few as $11 \%$ of inmates with SUDs receive treatment for addiction during their incarceration with an emphasis instead on education, detoxification, and abstinence $[28,40]$. Perhaps as a result, released prisoners are at extremely high 
risk for relapse to drugs or alcohol [41-43] with associated drug-related recidivism to prison [44] and excess mortality due to drug overdose $[45,46]$.

Treatment of SUDs is clearly beneficial for relapse prevention but may also improve engagement in HIV care. Indeed, two recent interventions $[33,47]$ have demonstrated that opioid agonist therapies initiated upon release are effective at preventing relapse to opioid use and secondarily maintain HAART-related benefits achieved during incarceration. Since persistent virologic suppression is associated with decreased risk of HIV transmission, treatment of SUDs might, indirectly, be an effective secondary prevention measure for HIV $[48,49]$. Treatment of SUDs is necessary but may be insufficient alone because of the many other complex social and behavioral stressors that simultaneously disrupt care in this patient population $[28,50]$. These will be addressed systematically in the following sections.

\subsection{Comorbid Mental Illness}

3.2.1. Epidemiology of the Problem. Substance use disorders are often concurrent and synergistic with mental health disorders and it may be difficult to disentangle the two issues. Over three-quarters of jail inmates with mental health disorders meet criteria for substance dependence or abuse [51]. Mental illness is highly prevalent in the CJS overall with $14-24 \%$ of prison and jail inmates reporting recent mental health problems [51]. Prevalence of psychiatric disorders is even higher among female compared to male inmates [32, 51]. The convergence of mental health disorders and HIV is especially evident in the CJS. Among inmates in the Texas Department of Criminal Justice, psychiatric disorders were more common among PLWHA compared to their uninfected peers [52].

3.2.2. Areas for Future Empirical Research. Little is known about the effects of mental health disorders on HIV outcomes in dually diagnosed persons in the CJS. In nonCJS settings, however, treatment of psychiatric comorbidities has a profound impact on adherence to antiretroviral therapy. Several studies have examined the association of depression with HAART nonadherence in community-based cohorts. Among HIV-infected women, depression has been associated with decreased virologic response to HAART, higher likelihood of immunologic failure, and higher risk for all-cause mortality [53]. The longitudinal Multicenter AIDS Cohort Study of men who have sex with men showed that depression increased risk of interrupting or discontinuing HAART [54].

Furthermore, relatively few studies have examined associations between serious mental illness (SMI) and HAART adherence despite significant epidemiologic overlap between HIV and SMI. SMI includes the diagnoses of schizophrenia, schizoaffective disorder, bipolar depression, and major depression with psychotic features. Adherence to psychiatric medications among SMI patients ranges between 40-60\%, suggesting that adherence to HAART in these populations might also be problematic [55]. This has especially troubling implications in the CJS where both HIV and SMI are highly concentrated. Approximately $14-22 \%$ of inmates in state and federal prisons/jails meet criteria for mania and $8-17 \%$ meet criteria for a psychotic disorder [51]. Interestingly, in one study of 47 community-based participants with SMI and HIV in Los Angeles, the mean adherence rate to antiretrovirals (proportion of prescribed doses taken) was $66 \%$ with surprisingly almost half of participants having a $>90 \%$ adherence rate to HAART. The authors note that this signifies that HAART adherence rates are similar to adherence rates among people living with HIV but without SMI in community clinics, implying that diagnosis of comorbid SMI should not preclude HIV treatment [55]. This, however, was only one small study of community-based patients with comorbid SMI and HIV. To our knowledge, there are no published studies examining antiretroviral or psychiatric medication adherence among subjects with both SMI and HIV in the CJS. Given the large overlap between HIV and mental health disorders in this setting, more research is needed on correlates of HAART adherence in the correctional population with dual diagnoses.

3.2.3. Areas for Future Interventional Research. Management of mental illness in correctional settings is an understudied but critical issue for people living with HIV. Among depressed HIV-infected patients in a non-correctional managed care setting, those who were treated with selective serotonin reuptake inhibitors for depression were more likely to be $>95 \%$ adherent to HAART medications than those with untreated depression [56]. In addition, depressed patients adherent to psychiatric medications had similar HAART adherence and HAART-related outcomes as non-depressed people living with HIV on HAART [56]. Another study of injection drug users enrolled in a trial of directly administered antiretroviral therapy found that improvements in depressive symptoms were associated with increased HAART adherence and increases in CD4 cell counts [57]. Treatment of depression and serious mental illness can lead to improved HIV outcomes and HAART adherence rates that reach parity with people living with HIV but without mental illness $[55,56]$.

Unfortunately, even if inmates are engaged in psychiatric care during incarceration, treatment of mental illness in the CJS is often disrupted after release to communities. Upon initial release, suicide is common among former inmates, ranking among the top 5 causes of death in one study of released prisoners in Washington state and indicating inadequate treatment of mental health disorders during this critical transition period [45]. System-based structural barriers to care may be seemingly insurmountable. Understaffing of outpatient psychiatric clinics and insurer-based restrictions on mental health coverage can lead to the unavailability of treatment for mentally ill persons in the community. This is of grave concern for mentally ill patients with HIV because, as aforementioned, undertreated mental illness is related to decreased HAART adherence, worse biological outcomes, and increased risk of death $[45,53,54,57]$. In addition, psychiatric disorders are highly associated with 
prison recidivism among both HIV-infected and uninfected individuals $[21,51,58]$.

Methods to coordinate psychiatric services upon release from correctional settings are necessary. Formal case management services that enhance linkages to psychiatric care may secondarily improve adherence to HAART, and research is currently ongoing in this field [59]. Other strategies of diverting mentally ill offenders to treatment rather than incarceration through the use of mental health courts have been shown successful in engaging individuals in psychiatric care and cost savings compared to incarceration. The impact of these programs on HIV outcomes is not known but would be an important area of interest in future interventional studies $[58,60]$.

\subsection{Homelessness}

3.3.1. Epidemiology of the Problem. A relative lack of mental health services in the community combined with the "War on Drugs" has resulted in the shuttling of mentally ill persons between homelessness and incarceration. Incarceration increases the risk of homelessness through loss of employment, loss of housing, and disruption of social support or community resources [61, 62]. Conversely, homelessness increases incarceration risk through common behaviors, including substance use and transactional sex [61-65]. Homelessness itself is also frequently criminalized, such that public intoxication, loitering, and vagrancy are behaviors punishable by incarceration. Approximately $23-68 \%$ of homeless individuals have a history of incarceration [61, 63]. Prevalence of homelessness prior to incarceration is twice as high among those with mental health problems as those without (13.2\% versus $6.3 \%$ in state prisons) [51]. As a result, a revolving door between homelessness and incarceration is common, especially among those with mental illness or substance abuse issues $[58,61,66]$.

Among PLWHA, homelessness and unstable housing have been associated with poor HIV outcomes including worse adherence to HAART, fewer ambulatory care visits, and increased risk of death [67-70]. A case-control study of the impact of housing on survival of persons with AIDS noted that $9.8 \%$ of persons were homeless at time of AIDS diagnosis and that 5 -year survival was far worse for those who were homeless compared to those who were housed $(67 \%$ versus $81 \%$, resp.) [69]. This study also noted that antiretroviral use was lower among the homeless compared to the housed and that provision of housing improved survival [69]. In Chicago, provision of housing to HIV-infected homeless subjects with recent hospitalizations improved AIDSfree survival at 12 months [67]. Similarly, a prospective three-city study (in Baltimore, Chicago, and Los Angeles) randomized homeless or unstably housed PLWHA to immediate enhanced rental assistance versus customary housing services. In an intention to treat analysis, there was no difference between the two groups in terms of adherence to HAART, HIV RNA levels, or CD4 lymphocyte counts. In an "as-treated" analysis; however, those who remained homeless at followup were significantly more likely to have a higher HIV RNA viral load, regardless of randomization group
[70]. Homelessness or unstable housing can have a profound impact on HAART adherence because homeless individuals are often distracted by trying to meet basic needs of food and shelter. The homeless often have difficulty safely storing medications although few current first-line antiretroviral regimens require refrigeration. Nevertheless, HAART may be successfully initiated in homeless or marginally housed patients: the three-city study did find that $78 \%$ of the homeless or unstably housed self-reported $100 \%$ past 2 -day adherence to HAART [71].

The effects of homelessness on HAART adherence are often confounded by the presence of comorbid mental illness or SUDs. Homeless or unstably housed individuals are less likely to be adherent to HAART if they have recent substance use or higher depression scores [71]. A randomized controlled trial of HIV-infected homeless or marginally housed adults with depression found that providing antidepressants increased probability of achieving HIV virologic suppression [72]. Thus, treatment of HIV among the homeless or marginally housed can be complicated by comorbid substance use or mental health issues but proactive treatment of these comorbid conditions can improve HIV outcomes.

3.3.2. Areas for Future Empirical Research. Although these studies have demonstrated the negative impact of homelessness on HAART adherence, there is no scientific literature concerning the impact of homelessness on recently incarcerated, HIV-infected populations. Given the high prevalence of homelessness after release from prison and the demonstrated beneficial effects of housing on social, psychiatric, addiction and HIV-related outcomes, future research should focus on correlates of risk of homelessness among recently-released prisoners and jail detainees.

Obtaining housing constitutes a top priority often expressed by HIV-infected released prisoners. Many prisoners return to communities plagued by poverty, unemployment, and violence- the same communities from which they were incarcerated. They must attempt to navigate this environment while simultaneously managing the more personal stresses of reuniting with parents, partners, and children after incarceration. Ideally, these relationships would support positive health promotion. All too often, however, these relationships are afflicted with addiction and interpersonal violence that only exacerbate stress and interfere with health-seeking behaviors. Returning prisoners must also attempt to manage comorbid medical illnesses, like diabetes and hypertension, which can become immediately life-threatening when undertreated, especially in the context of homelessness. These overlapping stresses (poverty, neighborhood characteristics, interpersonal relationships, and comorbid chronic medical conditions) may be important to individuals' health after prison release and likely contribute to prison recidivism. Unfortunately, there have been few published studies to date around homelessness and HIV-related continuity of care after release, thus limiting our discussion on these topics. Future studies should address the impact of homelessness and social instability, as more broadly defined, on healthcare utilization patterns of PLWHA after release from prison. 
3.3.3. Areas for Future Interventional Research. Despite the strong association between homelessness and incarceration, strategies addressing the housing needs of HIV-infected inmates upon release have not been well studied. A possible strategy that may improve comorbid substance use or psychiatric disorders as well as recidivism is a Housing First approach. Contrary to traditional substance abuse or mental health treatment programs which require strict adherence to treatment in order to maintain housing, Housing First programs provide immediate access to permanent housing without any prerequisites for psychiatric treatment or sobriety. Among homeless or marginally housed persons with serious mental illness, Housing First programs have been successful in improving residential status and decreasing use of inpatient, emergency, and criminal justice system services $[73,74]$. Although Housing First models have not been studied in the HIV-infected recently incarcerated population, its successes among vulnerable populations with serious mental illness or substance use disorders suggest it may be a promising strategy for this group.

Other possible interventions to improve HIV outcomes among homeless or marginally housed releasees involve improving engagement to care through increased supportive services. In San Francisco, a clinic specializing in supporting recently released inmates in the transition back to the community was noted to successfully engage a population in which $38 \%$ were homeless [75]. The clinic employed a full-time community health worker who attended parole meetings and provide enhanced case management services for clinic patients. Although the San Francisco clinic targeted non-HIV-infected persons, the transitions clinic may serve as a model for maintaining HIV-infected, homeless individuals in care. Currently, the HRSA-funded Enhancing Linkages to HIV Primary Care and Services in Jail Settings Initiative is studying the impact of enhanced case management services that can include housing services [59]. Although enhanced case management services may not improve linkage to care in all settings, [76] its efficacy among homeless populations has not yet been studied.

Fortunately, homelessness is a modifiable risk factor that could be prevented with effective discharge planning services. Transitional case management programs should proactively address housing instability as part of a comprehensive package of social services, recognizing the extreme vulnerability during this period of movement between prisons and communities. Provision of housing is, like many of the other issues addressed here, not a stand-alone problem.

\subsection{Loss of Medical and Social Benefits}

3.4.1. Epidemiology of the Problem. In the current USA healthcare system, where medical care is predominantly funded by third-party payors, gaps in insurance coverage during incarceration contribute to fragmentation of care upon release. By virtue of their socioeconomic or medical disability status, most inmates with health insurance have Medicaid prior to incarceration. Medicaid prohibits any use of federal funds for medical or psychiatric care of inmates in jails and prisons. Most states (>90\%) terminate Medicaid coverage during incarceration [77]. For those who qualify for Medicaid by virtue of their receiving social security income, termination, rather than suspension, of benefits is almost guaranteed [77, 78]. Termination signifies that inmates are completely removed from Medicaid rolls and are required to reapply. Because of the difficulties former inmates face in obtaining employment, employment-based health insurance is not a reliable option. In addition, job applications for even the most menial opportunities often require a permanent address, which many former inmates cannot provide [79]. During incarceration, many other social benefits including food stamps, state-subsidized housing, and temporary assistance for needy families are also suspended or discontinued [80].

As a result, many inmates are left with gaps in medical and social benefit coverage upon release from prison. Reapplication procedures for Medicaid can be initiated as early as 45-90 days prior to expected release [78] but require photo identification and other documentation that are sometimes logistically difficult for former inmates to obtain. Furthermore, released prisoners often are returned to communities late at night or on weekends when services are closed [79]. Because of these barriers, a survey of 511 women leaving New York's jails found that only half of the participants had obtained health insurance by one year after release, regardless of whether or not they had comorbid conditions like asthma, diabetes, or HIV that required ongoing medical care [81]. Interruptions in coverage result in a greater reliance on "free" episodic emergency and hospital-based care [82] and decreased engagement with mental health services for the mentally ill [83]. Perhaps because having medical insurance is a marker of social stability, subjects who obtained health insurance coverage following release were $69 \%$ less likely to be rearrested for any cause and $91 \%$ less likely to be rearrested on drug-related charges at the time of follow-up compared to those without health insurance [44].

For HIV-infected released prisoners, continuity of medical coverage is especially crucial for continued provision of HAART. In the past, coverage for antiretrovirals was dismal: in a 1994 study, 19\% of HIV-infected prisoners reported trying to get rearrested just to obtain prison-based HIV care that was seemingly not available to them in the community after release [84]. Fortunately today, coverage gaps are bridged by the AIDS Drug Assistance Program (ADAP) which, under HRSA's Ryan White HIV/AIDS Treatment Modernization Act Part B, acts as the "payor of last resort." [85] Baillargeon et al. [22] estimated that $100 \%$ of HIV-infected inmates who received HAART during incarceration in Texas would qualify for ADAP. Over the past decade, ADAP has made significant inroads into covering this vulnerable population.

3.4.2. Areas for Future Empirical Research. Beyond the availability of medication assistance programs, there remains a significant knowledge gap about why and how released prisoners experience decreased persistence in HIV care. Certainly the problem is multifactorial and complex: this population is often marginalized from the mainstream medical community because of active drug use, mental illness, 
or HIV-related stigma prior to and following incarceration. Even in Canada, in which a nationalized healthcare system removes significant insurance-related barriers to care, released prisoners report problems obtaining appointments with an HIV clinician after return to the community [86]. These appointment delays have been associated with lapses in medication adherence lasting 1-3 days which, especially in the case of antiretrovirals with low genetic barriers to resistance, may be sufficient to result in virologic failure. Other lapses in HAART adherence have been specifically linked to points of custody transfers (i.e., movement from police stations or court to correctional facilities, transfer from prisons to communities.) [86]. Future research around correlates of decreased persistence in HIV care for released prisoners should incorporate mixed methods with qualitative interviews of released prisoners to increase granularity of available data.

3.4.3. Areas for Future Interventional Research. In spite of significant safety nets for people living with HIV, there remain gaps in medical coverage after release that present barriers to continuous HIV care. These should be addressed by future interventions. Delays in completing and processing ADAP applications are a limiting factor in HIV-infected recently released prisoners benefiting from the program. In a cross-sectional mixed methods study of 105 HIV-infected recently released prisoners in Florida, 19\% reported that the paperwork required to obtain medical care, insurance, and HAART presented a major obstacle to care [79]. Of 177 HIVinfected reincarcerated jail inmates, $48 \%$ had no health insurance and only $61 \%$ had ADAP when living in the community [34]. Of those who reported taking HAART in the community, $19 \%$ never once saw an HIV provider during the 12-month-observation period [34]. Conversely, inmates who received assistance with ADAP applications in Texas prior to release were three times more likely to fill HAART prescriptions within 10 days after release [22]. This latter study in Texas was one of the few to date that incorporated assistance with ADAP applications into a comprehensive transitional case management program. $\mathrm{Fu}-$ ture interventions should include similar programs while taking an evidence-based approach to measuring important outcomes, including HAART adherence, continuity of care, enrollment in an HIV clinic, prison recidivism, and HIVassociated morbidity.

\section{Summary}

Each year, almost 10 million people are released from USA jails and prisons to the community [3, 44]. A small but significant proportion of these released prisoners are living with HIV, a disease best managed with lifelong combination antiretroviral therapy in regular consultation with an HIV specialist. Continuity of care is critical for prevention of HIV-related morbidity and mortality but faces numerous obstacles in the transitions from community to prison and back to the community. In this paper we explored four major challenges to successful management of HIV in correctional populations: relapse to substance use, mental illness, homelessness, and loss of medical and social benefits. Each of these areas constitutes a competing priority upon release that demands immediate attention and diverts time, energy, and scarce resources away from engagement in care. In this era of HAART, HIV may be perceived as a chronic, asymptomatic disease of less urgent interest to returning prisoners than meeting basic subsistence needs, coping with debilitating psychiatric illnesses or meeting the physical and mental demands of powerful addictions. These barriers to continuity of HIV care and HAART adherence must be addressed for the sake of the individual and from a public health standpoint.

The numerous outstanding knowledge gaps and solution gaps concerning HIV outcomes in criminal justice populations also present exciting academic opportunities for future research. We have attempted to shed light on these gaps in support of a contemporary research agenda. Evidencebased examinations of the problems and solutions facing criminal justice populations are critical to progress in the management of HIV in correctional systems.

\section{Discussion}

Our review of the literature suggests that linkages from prison-based to community HIV care are critical for maintaining the benefits of HAART achieved during incarceration. There is ongoing debate in the criminal justice field about how best to design transitional interventions to address these complex issues that otherwise disrupt care for returning prisoners. Options proposed thus far include intensive case management, supportive housing, opioid substitution therapy, directly administered antiretroviral therapy, medication adherence support, or combinations therein. We advocate that, regardless of methodology, the most effective interventions require significant breadth to address all of the barriers that manifest during transfer from prison to the community.

Unfortunately, there are significant logistical constraints to introducing evidence-based interventions into correctional systems either during incarceration or prior to release. Inmates are often transitory, cycling frequently and rapidly between jails and communities or among various correctional facilities. This migratory pattern provides a narrow window in which to introduce and assess any given intervention. Constraints may also be imposed by a correctional system itself that enforces strict privacy protection measures (especially regarding HIV serostatus) and is subject to state, or federally based budgetary limitations. Even outside prison walls, and thus, technically outside of correctional systems, released prisoners may be difficult subjects in longitudinal studies because they often experience high rates of attrition. Strategies for collaboration between academic centers of research and correctional systems have been recently proposed elsewhere [87].

Despite these challenges, empirical and interventional research is strongly needed to improve the management of HIV both during and following incarceration. Future research 
must work to develop geographically and culturally appropriate interventions that are highly tailored to individual needs. Perhaps most difficult, future research should attempt to deconstruct structural barriers to persistence in HIV care that include termination of medical/social benefits during incarceration, minimal linkages to community-based psychiatric and HIV primary care, and often-absent treatment for substance use disorders. As providers and clinical researchers, we must act as advocates for this often silenced and marginalized population living with HIV.

\section{Conflict of Interests}

The authors declare that there are no competing financial interests exist.

\section{Contribution}

Jaimie P. Meyer, Nadine E. Chen and Sandra A. Springer designed this project, researched, and wrote drafts of the paper. Jaimie P. Meyer integrated contributions and prepared the manuscript for submission. Sandra A. Springer edited the paper. All authors contributed to and approved the final version of the paper.

\section{Acknowledgments}

The authors would like to acknowledge career development funding from the National Institute of Mental Health (T32 MH020031 for Jaimie P. Meyer), National Institute on Drug Abuse (K23 DA019381 and R01 DA030762 for Sandra A. Springer), and the National Institute on Alcohol Abuse and Alcoholism (R01 AA018944 for Sandra A. Springer). The funding sources had no role in study design, data collection, analysis and interpretation of data, writing of the paper, or in the decision to submit the paper for publication.

\section{References}

[1] J. L. Farley, J. A. Mitty, M. A. Lally et al., "Comprehensive medical care among HIV-Positive incarcerated women: the Rhode Island experience," Journal of Women's Health and Gender-Based Medicine, vol. 9, no. 1, pp. 51-56, 2000.

[2] C. G. Beckwith, N. D. Zaller, J. J. Fu, B. T. Montague, and J. D. Rich, "Opportunities to diagnose, treat, and prevent HIV in the criminal justice system," Journal of Acquired Immune Deficiency Syndromes, vol. 55, supplement 1, pp. S49-S55, 2010.

[3] A. C. Spaulding, R. M. Seals, M. J. Page, A. K. Brzozowski, W. Rhodes, and T. M. Hammett, "HIV/AIDS among inmates of and releasees from US correctional facilities, 2006: declining share of epidemic but persistent public health opportunity," PLoS One, vol. 4, no. 11, Article ID e7558, 2009.

[4] D. S. Maru, S. Basu, and F. L. Altice, "HIV control efforts should directly address incarceration," The Lancet Infectious Diseases, vol. 7, no. 9, pp. 568-569, 2007.

[5] A. Palepu, M. W. Tyndall, K. Chan, E. Wood, J. S. G. Montaner, and R. S. Hogg, "Initiating highly active antiretroviral therapy and continuity of HIV care: the impact of incarceration and prison release on adherence and HIV treatment outcomes," Antiviral Therapy, vol. 9, no. 5, pp. 713-719, 2004.

[6] J. H. Samet, K. A. Freedberg, J. B. Savetsky, L. M. Sullivan, L. Padmanabhan, and M. D. Stein, "Discontinuation from HIV medical care: squandering treatment opportunities," Journal of Health Care for the Poor and Underserved, vol. 14, no. 2, pp. 244-255, 2003.

[7] L. Maruschak, "Bureau of Justice Statistics Bulletin: HIV in Prisons, 2007-2008," http://bjs.ojp.usdoj.gov/index.cfm?ty= pbdetail\&amp.

[8] S. Fazel and J. Baillargeon, "The health of prisoners," The Lancet, vol. 377, no. 9, pp. 956-965, 2010.

[9] D. W. Roberson, B. L. White, and C. I. Fogel, "Factors influencing adherence to antiretroviral therapy for HIV-infected female inmates," Journal of the Association of Nurses in AIDS Care, vol. 20, no. 1, pp. 50-61, 2009.

[10] F. Mostashari, E. Riley, P. A. Selwyn, and F. L. Altice, "Acceptance and adherence with antiretroviral therapy among HIVinfected women in a correctional facility," Journal of Acquired Immune Deficiency Syndromes and Human Retrovirology, vol. 18, no. 4, pp. 341-348, 1998.

[11] D. W. Seal, G. D. Eldridge, B. Zack, and J. Sosman, "HIV testing and treatment with correctional populations: people, not prisoners," Journal of Health Care for the Poor and Underserved, vol. 21, no. 3, pp. 977-985, 2010.

[12] N. Zaller, P. Thurmond, and J. D. Rich, "Limited spending: an analysis of correctional expenditures on antiretrovirals for HIV-infected prisoners," Public Health Reports, vol. 122, no. 1, pp. 49-54, 2007.

[13] J. Baillargeon, M. J. Borucki, S. Zepeda, H. B. Jenson, and C. T. Leach, "Antiretroviral prescribing patterns in the texas prison system," Clinical Infectious Diseases, vol. 31, no. 6, pp. 14761481, 2000.

[14] S. A. Springer, E. Pesanti, J. Hodges, T. Macura, G. Doros, and F. Altice, "Effectiveness of antiretroviral therapy among HIVinfected prisoners: reincarceration and the lack of sustained benefit after release to the community," Clinical Infectious Diseases, vol. 38, no. 12, pp. 1754-1760, 2004.

[15] S. A. Springer, G. H. Friedland, G. Doras, E. Pesanti, and F. Altice, "Antiretroviral treatment regimen outcomes among HIV-infected prisoners," HIV Clinical Trials, vol. 8, no. 4, pp. 205-212, 2007.

[16] S. S. Hatcher, I. A. Toldson, D. C. Godette, and J. B. Richardson, "Mental health, substance abuse, and HIV disparities in correctional settings: practice and policy implications for African Americans," Journal of Health Care for the Poor and Underserved, vol. 20, supplement 2, pp. 6-16, 2009.

[17] F. Altice, A. Kamarulzaman, V. Soriano, M. Schechter, and G. Friedland, "Treatment of medical, psychiatric, and substanceuse comorbidities in people infected with HIV who use drugs," The Lancet, vol. 376, no. 9738, pp. 367-387, 2010.

[18] Court USS, "Estelle v. Gamble," 1976.

[19] United States District Court District of Connecticut Doe v. Meachum. Vol. Civil Action No. H-88-562, 1990.

[20] B. L. Stephenson, D. A. Wohl, C. E. Golin, H. C. Tien, P. Stewart, and A. H. Kaplan, "Effect of release from prison and re-incarceration on the viral loads of HIV-infected individuals," Public Health Reports, vol. 120, no. 1, pp. 84-88, 2005.

[21] J. Baillargeon, T. Giordano, A. Harzke et al., "Predictors of reincarceration and disease progression among released HIVinfected inmates," AIDS Patient Care and STDs, vol. 24, no. 6, pp. 389-394, 2010.

[22] J. Baillargeon, T. Giordano, J. D. Rich et al., "Accessing antiretroviral therapy following release from prison," Journal 
of the American Medical Association, vol. 301, no. 8, pp. 848857, 2009.

[23] J. Baillargeon, T. Giordano, A. Harzke, G. Baillargeon, J. Rich, and D. Paar, "Enrollment in outpatient care among newly released prison inmates with HIV infection," Public Health Reports, vol. 125, supplement 1, pp. 64-71, 2010.

[24] P. Choi, R. Kavasery, M. M. Desai, S. Govindasamy, A. Kamarulzaman, and F. L. Altice, "Prevalence and correlates of community re-entry challenges faced by HIV-infected male prisoners in Malaysia," International Journal of STD \&AIDS, vol. 21, no. 6, pp. 416-423, 2010.

[25] N. P. Pai, M. Estes, E. E. Moodie, A. L. Reingold, and J. P. Tulsky, "The impact of antiretroviral therapy in a cohort of HIV infected patients going in and out of the San Francisco County jail," PLoS One, vol. 4, no. 9, Article ID e7115, 2009.

[26] W. Burman, B. Grund, J. Neuhaus et al., "Episodic antiretroviral therapy increases hiv transmission risk compared with continuous therapy: results of a randomized controlled trial," Journal of Acquired Immune Deficiency Syndromes, vol. 49, no. 2, pp. 142-150, 2008.

[27] L. Grinspoon and J. B. Bakalar, "The war on drugs-a peace proposal," The New England Journal of Medicine, vol. 330, no. 5, pp. 357-360, 1994.

[28] CASA, Behind Bars II: Substance Abuse and America's Prison Population, National Center on Addiction and Substance Abuse at Columbia University, New York, NY, USA, 2010.

[29] Arrestee Drug Abuse Monitoring Program II, Annual Report. Washington, DC, USA, 2009.

[30] J. C. Karberg and C. J. Mumola, "Drug use and dependence, state and federal prisoners," Bureau of Justice Statistics Special Report, NCJ 213530, Office of Justice Programs, US Department of Justice, Washington, DC, USA, 2007.

[31] S. Fazel, P. Bains, and H. Doll, "Substance abuse and dependence in prisoners: a systematic review," Addiction, vol. 101, no. 2, pp. 181-191, 2006.

[32] I. A. Binswanger, J. O. Merrill, P. M. Krueger, M. C. White, R. E. Booth, and J. G. Elmore, "Gender differences in chronic medical, psychiatric, and substance-dependence disorders among jail inmates," The American Journal of Public Health, vol. 100, no. 3, pp. 476-482, 2010.

[33] S. Springer, S. Chen, and F. Altice, "Improved HIV and substance abuse treatment outcomes for released HIV-infected prisoners: the impact of buprenorphine treatment," Journal of Urban Health, vol. 87, no. 4, pp. 592-602, 2010.

[34] K. Clements-Nolle, R. Marx, M. Pendo, E. Loughran, M. Estes, and M. Katz, "Highly active antiretroviral therapy use and HIV transmission risk behaviors among individuals who are HIV infected and were recently released from jail," The American Journal of Public Health, vol. 98, no. 4, pp. 661-666, 2008.

[35] A. Palepu, M. W. Tyndall, K. Li et al., "Alcohol use and incarceration adversely affect HIV-1 RNA suppression among injection drug users starting antiretroviral therapy," Journal of Urban Health, vol. 80, no. 4, pp. 667-675, 2003.

[36] A. Harzke, M. Ross, and D. Scott, "Predictors of post-release primary care utilization among HIV-positive prison inmates: a pilot study," AIDS Care, vol. 18, no. 4, pp. 290-301, 2006.

[37] M. Azar, S. Springer, J. Meyer, and F. Altice, "A systematic review of the impact of alcohol use disorders on HIV treatment outcomes, adherence to antiretroviral therapy and health care utilization," Drug Alcohol Depend, vol. 112, no. 3, pp. 178-193, 2010.

[38] G. M. Lucas, "Substance abuse, adherence with antiretroviral therapy, and clinical outcomes among HIV-infected individuals," Life Science, vol. 88, no. 21-22, pp. 948-952, 2010.
[39] J. D. Rich, A. E. Boutwell, D. C. Shield et al., "Attitudes and practices regarding the use of methadone in US state and federal prisons," Journal of Urban Health, vol. 82, no. 3, pp. 411-419, 2005.

[40] H. K. Wexler and B. W. Fletcher, "National criminal justice drug abuse treatment studies overview," The Prison Journal, vol. 87, no. 1, pp. 9-24, 2007.

[41] J. van Olphen, N. Freudenberg, P. Fortin, and S. Galea, "Community reentry: perceptions of people with substance use problems returning home from New York City jails," Journal of Urban Health, vol. 83, no. 3, pp. 372-381, 2006.

[42] L. Harrison, "The revolving prison door for drug-involved offenders: challenges and opportunities," Crime \& Delinquency, vol. 47, no. 3, pp. 462-485, 2001.

[43] R. K. Chandler, B. W. Fletcher, and N. D. Volkow, "Treating drug abuse and addiction in the criminal justice system: improving public health and safety," Journal of the American Medical Association, vol. 301, no. 2, pp. 183-190, 2009.

[44] N. Freudenberg, J. Daniels, M. Crum, T. Perkins, and B. E. Richie, "Coming home from jail: the social and health consequences of community reentry for women, male adolescents, and their families and communities," T.he American Journal of Public Health, vol. 98, no. 9, pp. S191-S202, 2008.

[45] I. A. Binswanger, M. F. Stern, R. A. Deyo et al., "Release from prison-a high risk of death for former inmates," The New England Journal of Medicine, vol. 356, no. 2, pp. 157-165, 2007.

[46] D. L. Rosen, V. J. Schoenbach, and D. A. Wohl, "All-cause and cause-specific mortality among men released from state prison, 1980-2005," The American Journal of Public Health, vol. 98, no. 12, pp. 2278-2284, 2008.

[47] J. D. Rich, M. McKenzie, D. C. Shield et al., "Linkage with methadone treatment upon release from incarceration: a promising opportunity," Journal of Addictive Diseases, vol. 24, no. 3, pp. 49-59, 2005.

[48] T. Hollingsworth, O. Laeyendecker, G. Shirreff et al., "HIV1 transmitting couples have similar viral load set-points in Rakai, Uganda," PLoS Pathogens, vol. 6, no. 5, Article ID e1000876, pp. 1-9, 2010.

[49] D. Wilson, M. Law, A. Grulich, D. Cooper, and J. Kaldor, "Relation between HIV viral load and infectiousness: a modelbased analysis," The Lancet, vol. 372, no. 9635, pp. 314-320, 2008.

[50] S. Belenko, "Assessing released inmates for substance abuserelated service needs," Crime \& Delinquency, vol. 52, no. 1, pp. 94-113, 2006.

[51] D. J. James and L. E. Glaze, "Mental health problems of prison and jail inmates," Bureau of Justice Statistics Special Report, NCJ 213600, Office of Justice Programs, US Department of Justice, Washington, DC, USA, 2006, http://bjs .ojp.usdoj.gov/index.cfm?ty=pbdetail\&iid $=789$.

[52] J. G. Baillargeon, D. P. Paar, H. Wu et al., "Psychiatric disorders, HIV infection and HIV/hepatitis co-infection in the correctional setting," AIDS Care, vol. 20, no. 1, pp. 124-129, 2008.

[53] K. Anastos, M. F. Schneider, S. J. Gange et al., "The association of race, sociodemographic, and behavioral characteristics with response to highly active antiretroviral therapy in women," Journal of Acquired Immune Deficiency Syndromes, vol. 39, no. 5, pp. 537-544, 2005.

[54] X. Li, J. B. Margolick, C. S. Conover et al., "Interruption and discontinuation of highly active antiretroviral therapy in the multicenter AIDS cohort study," Journal of Acquired Immune Deficiency Syndromes, vol. 38, no. 3, pp. 320-328, 2005. 
[55] G. J. Wagner, D. E. Kanouse, P. Koegel, and G. Sullivan, "Adherence to HIV antiretrovirals among persons with serious mental illness," AIDS Patient Care and STDs, vol. 17, no. 4, pp. 179-186, 2003.

[56] M. A. Horberg, M. J. Silverberg, L. B. Hurley et al., "Effects of depression and selective serotonin reuptake inhibitor use on adherence to highly active antiretroviral therapy and on clinical outcomes in HIV-infected patients," Journal of Acquired Immune Deficiency Syndromes, vol. 47, no. 3, pp. 384390, 2008.

[57] S. A. Springer, S. Chen, and F. Altice, "Depression and symptomatic response among HIV-infected drug users enrolled in a randomized controlled trial of directly administered antiretroviral therapy," AIDS Care, vol. 21, no. 8, pp. 976-983, 2009.

[58] J. Baillargeon, I. A. Binswanger, J. V. Penn, B. A. Williams, and O. J. Murray, "Psychiatric disorders and repeat incarcerations: the revolving prison door," American Journal of Psychiatry, vol. 166, no. 1, pp. 103-109, 2009.

[59] J. Draine, D. Ahuja, F. L. Altice et al., "Strategies to enhance linkages between care for HIV/AIDS in jail and community settings," AIDS Care, vol. 23, no. 3, pp. 366-377, 2011.

[60] B. M. Kuehn, "Mental health courts show promise," Journal of the American Medical Association, vol. 297, no. 15, pp. 1641$1643,2007$.

[61] M. B. Kushel, J. A. Hahn, J. L. Evans, D. R. Bangsberg, and A. R. Moss, "Revolving doors: imprisonment among the homeless and marginally housed population," The American Journal of Public Health, vol. 95, no. 10, pp. 1747-1752, 2005.

[62] J. van Olphen, M. J. Eliason, N. Freudenberg, and M. Barnes, "Nowhere to go: how stigma limits the options of female drug users after release from jail," Substance Abuse Treatment, Prevention, and Policy, vol. 4, p. 10, 2009.

[63] C. Courtenay-Quirk, S. L. Pals, D. P. Kidder, K. Henny, and J. G. Emshoff, "Factors associated with incarceration history among HIV-positive persons experiencing homelessness or imminent risk of homelessness," Journal of Community Health, vol. 33, no. 6, pp. 434-443, 2008.

[64] D. P. Kidder, R. J. Wolitski, S. L. Pals, and M. L. Campsmith, "Housing status and HIV risk behaviors among homeless and housed persons with HIV," Journal of Acquired Immune Deficiency Syndromes, vol. 49, no. 4, pp. 451-455, 2008.

[65] C. I. Cohen, J. Sokolovsky, and M. Crane, "Aging, homelessness, and the law," International Journal of Law and Psychiatry, vol. 24, no. 2-3, pp. 167-181, 2001.

[66] D. Michaels, S. R. Zoloth, P. Alcabes, C. A. Braslow, and S. Safyer, "Homelessness and indicators of mental illness among inmates in New York City's correctional system," Hospital and Community Psychiatry, vol. 43, no. 2, pp. 150-155, 1992.

[67] D. Buchanan, R. Kee, L. S. Sadowski, and D. Garcia, "The health impact of supportive housing for HIV-positive homeless patients: a randomized controlled trial," The American Journal of Public Health, vol. 99, supplement 3, pp. S675-S680, 2009.

[68] D. P. Kidder, R. J. Wolitski, M. L. Campsmith, and G. V. Nakamura, "Health status, health care use, medication use, and medication adherence among homeless and housed people living with HIV/AIDS," The American Journal of Public Health, vol. 97, no. 12, pp. 2238-2245, 2007.

[69] S. K. Schwarcz, L. C. Hsu, E. Vittinghoff, A. Vu, J. D. Bamberger, and M. H. Katz, "Impact of housing on the survival of persons with AIDS," British Medical Public Health, vol. 9, p. 220, 2009.
[70] R. J. Wolitski, D. P. Kidder, S. L. Pals et al., "Randomized trial of the effects of housing assistance on the health and risk behaviors of homeless and unstably housed people living with HIV," AIDS and Behavior, vol. 14, no. 3, pp. 493-503, 2010.

[71] S. W. Royal, D. P. Kidder, S. Patrabansh et al., "Factors associated with adherence to highly active antiretroviral therapy in homeless or unstably housed adults living with HIV," AIDS Care, vol. 21, no. 4, pp. 448-455, 2009.

[72] A. C. Tsai, S. D. Weiser, M. L. Petersen, K. Ragland, M. B. Kushel, and D. R. Bangsberg, "A marginal structural model to estimate the causal effect of antidepressant medication treatment on viral suppression among homeless and marginally housed persons with HIV," Archives of General Psychiatry, vol. 67, no. 12, pp. 1282-1290, 2010.

[73] T. P. Gilmer, A. Stefancic, S. L. Ettner, W. G. Manning, and S. Tsemberis, "Effect of full-service partnerships on homelessness, use and costs of mental health services, and quality of life among adults with serious mental illness," Archives of General Psychiatry, vol. 67, no. 6, pp. 645-652, 2010.

[74] S. Tsemberis, L. Gulcur, and M. Nakae, "Housing first, consumer choice, and harm reduction for homeless individuals with a dual diagnosis," The American Journal of Public Health, vol. 94, no. 4, pp. 651-656, 2004.

[75] E. A. Wang, C. S. Hong, L. Samuels, S. Shavit, R. Sandersa, and M. Kushel, "Transitions clinic: creating a communitybased model of health care for recently released California prisoners," Public Health Reports, vol. 125, no. 2, pp. 171-177, 2010.

[76] D. A. Wohl, A. Scheyett, C. E. Golin et al., "Intensive case management before and after prison release is no more effective than comprehensive pre-release discharge planning in linking HIV-infected prisoners to care: a randomized trial," AIDS and Behavior, vol. 2011, no. 2, pp. 356-364, 2011.

[77] S. E. Wakeman, M. E. McKinney, and J. D. Rich, "Filling the gap: the importance of medicaid continuity for former inmates," Journal of General Internal Medicine, vol. 24, no. 7, pp. 860-862, 2009.

[78] L. M. Pérez, M. J. Ro, and H. M. Treadwell, "Vulnerable populations, prison, and federal and state Medicaid policies: avoiding the loss of a right to care," Journal of Correctional Health Care, vol. 15, no. 2, pp. 142-149, 2009.

[79] L. Fontana and A. Beckerman, "Recently released with HIV/AIDS: primary care treatment needs and experiences," Journal of Health Care for the Poor and Underserved, vol. 18, no. 3, pp. 699-714, 2007.

[80] Bazelon Center, "Arrested? What happens to your benefits if you go to jail or prison?" Judge David L. Bazelon Center for Mental Health Law, 2010, http://www.bazelon.org/News-Publications/Publications/CategoryID/7/List/1/Level/a/ProductID/21.aspx?SortField=ProductNumber\%2cProductNumber.

[81] J. Lee, D. Vlahov, and N. Freudenberg, "Primary care and health insurance among women released from New York City jails," Journal of Health Care for the Poor and Underserved, vol. 17, no. 1, pp. 200-217, 2006.

[82] A. B. Bindman, A. Chattopadhyay, and G. M. Auerback, "Interruptions in medicaid coverage and risk for hospitalization for ambulatory care-sensitive conditions," Annals of Internal Medicine, vol. 149, no. 12, pp. 854-860, 2008.

[83] J. P. Morrissey, H. J. Steadman, K. M. Dalton, A. Cuellar, P. Stiles, and G. S. Cuddeback, "Medicaid enrollment and mental health service use following release of jail detainees with severe mental illness," Psychiatric Services, vol. 57, no. 6, pp. 809-815, 2006 
[84] N. Warren, E. Bellin, S. Zoloth, and S. Safyer, "Human immunodeficiency virus infection care is unavailable to inmates on release from jail," Archives of Family Medicine, vol. 3, no. 10, pp. 894-898, 1994.

[85] Health Resources and Services Administration (HRSA), "Ryan White HIV/AIDS program," http://hab.hrsa.gov/.

[86] W. Small, E. Wood, G. Betteridge, J. Montaner, and T. Kerr, "The impact of incarceration upon adherence to HIV treatment among HIV-positive injection drug users: a qualitative study," AIDS Care, vol. 21, no. 6, pp. 708-714, 2009.

[87] J. D. Rich, D. Wohl, C. G. Beckwith et al., "HIV-related research in correctional populations: now is the time," Current HIV/AIDS Reports. In press. 


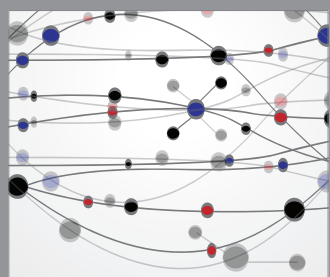

The Scientific World Journal
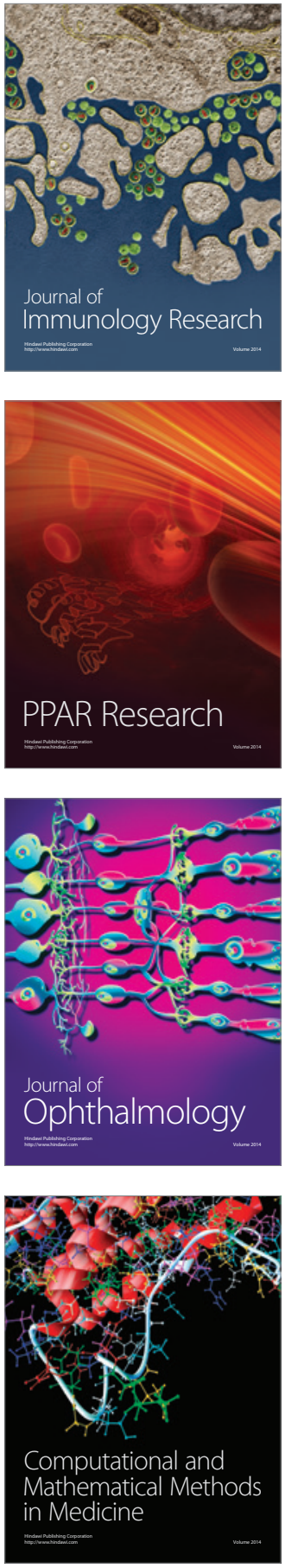

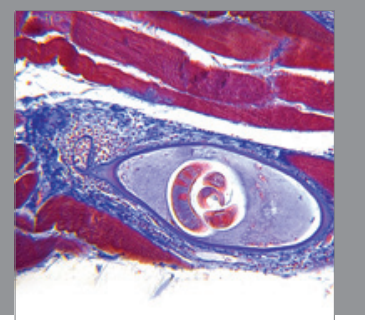

Gastroenterology

Research and Practice
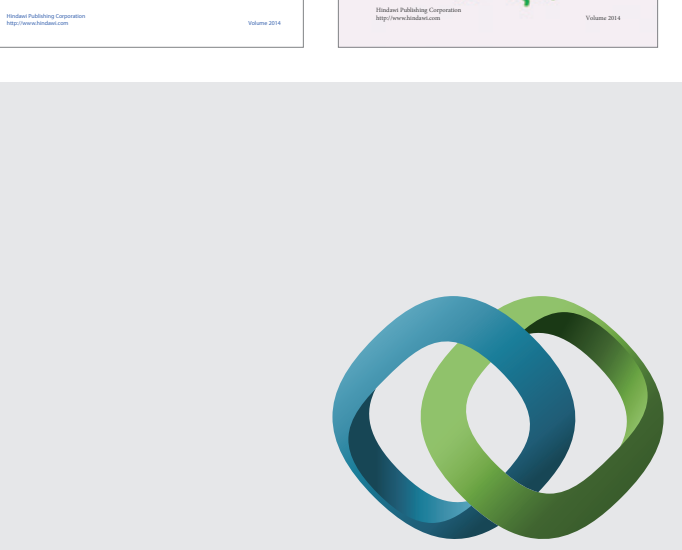

\section{Hindawi}

Submit your manuscripts at

http://www.hindawi.com
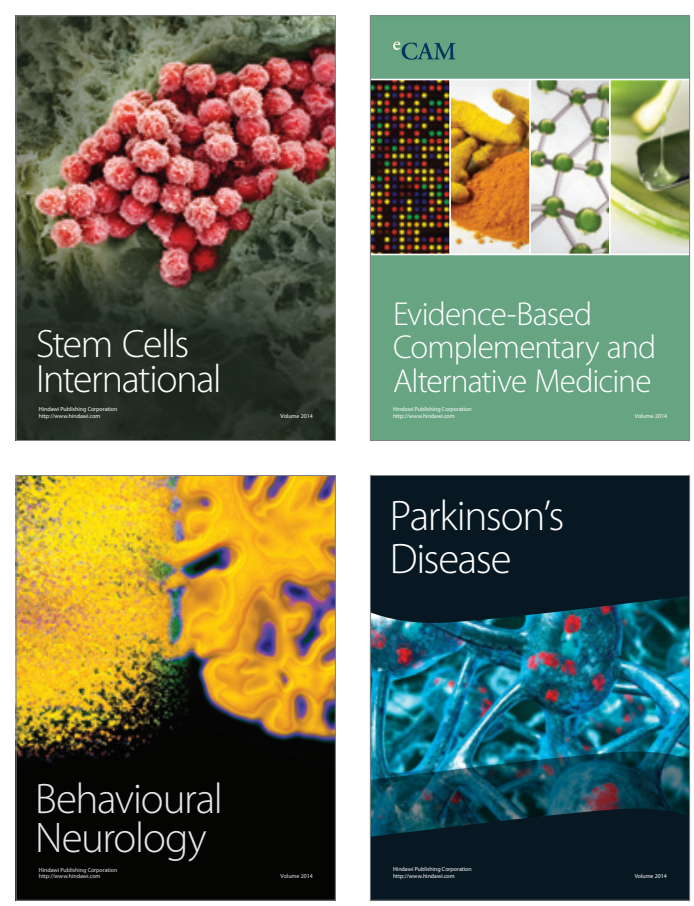

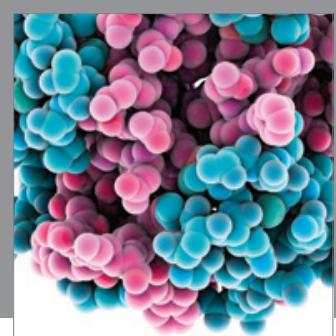

Journal of
Diabetes Research

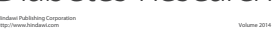

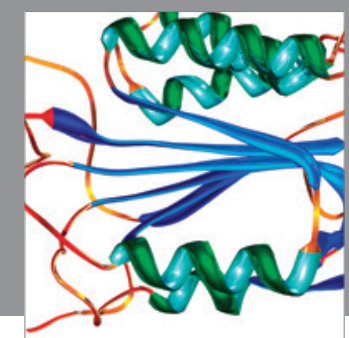

Disease Markers
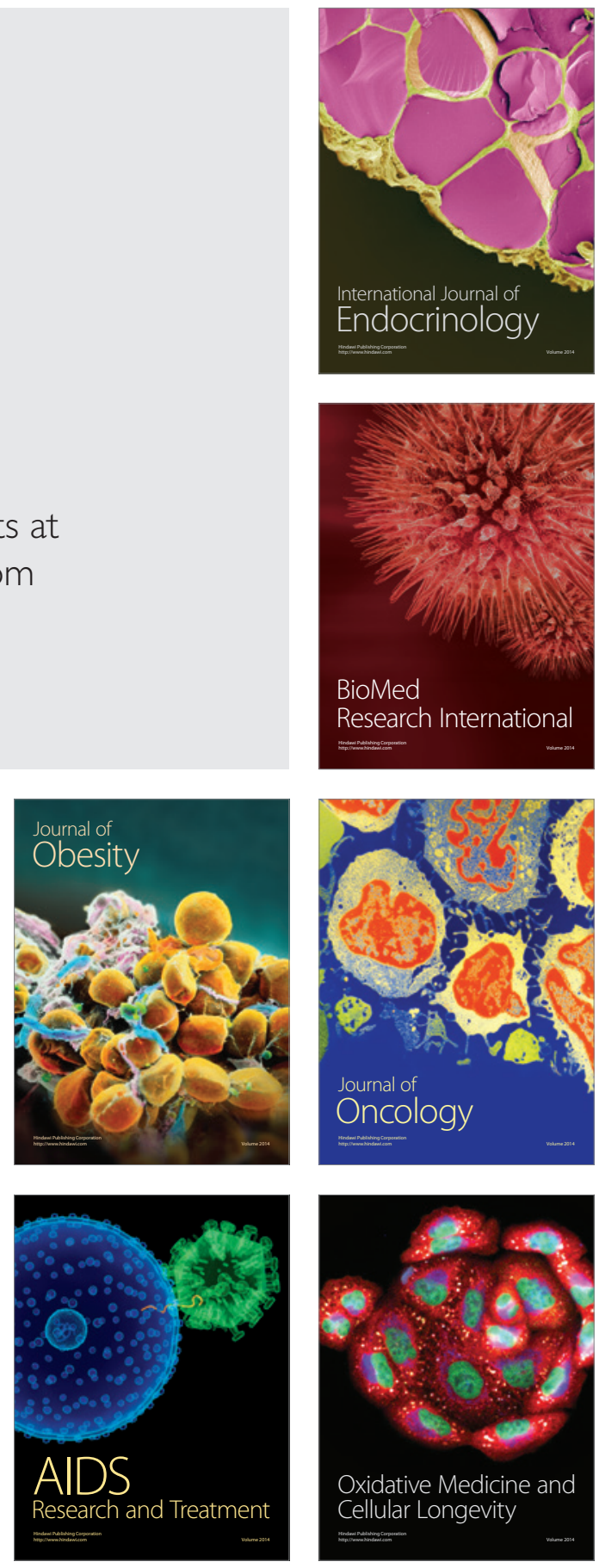